OPEN ACCESS

Edited by:

Olga Matos,

New University of Lisbon, Portugal

Reviewed by:

Walter Mazzucco,

University of Palermo, Italy

Mengyan Wang,

Hangzhou Xixi Hospital, China

${ }^{*}$ Correspondence:

Enrique J. Calderón

sandube@cica.es

Specialty section:

This article was submitted to

Epidemiology,

a section of the journal

Frontiers in Public Health

Received: 27 July 2019 Accepted: 27 September 2019

Published: 15 October 2019

Citation:

Morilla R, González-Magaña A, Friaza V, de Armas Y, Medrano FJ,

Calderón EJ and de la Horra C (2019)

Genetic Polymorphisms of Superoxide

Dismutase Locus of Pneumocystis

jirovecii in Spanish Population.

Front. Public Health 7:292

doi: 10.3389/fpubh.2019.00292

\section{Genetic Polymorphisms of Superoxide Dismutase Locus of Pneumocystis jirovecii in Spanish Population}

\author{
Rubén Morilla ${ }^{1,2,3}$, Amaia González-Magaña ${ }^{2,3}$, Vicente Friaza ${ }^{4}$, Yaxsier de Armas ${ }^{4}$, \\ Francisco J. Medrano ${ }^{2,3}$, Enrique J. Calderón ${ }^{2,3 *}$ and Carmen de la Horra ${ }^{2,3}$ \\ ${ }^{1}$ Department of Nursing, Universidad de Sevilla, Seville, Spain, ${ }^{2}$ Instituto de Biomedicina de Sevilla, Hospital Universitario \\ Virgen del Rocío, Consejo Superior de Investigaciones Científicas, Universidad de Sevilla, Seville, Spain, ${ }^{3}$ Centro de \\ Investigación Biomédica en Red de Epidemiología y Salud Pública (CIBERESP), Hospital Universitario Virgen del Rocío, \\ Seville, Spain, ${ }^{4}$ Hospital Microbiology Department, Institute of Tropical Medicine "Pedro Kouri", Havana, Cuba
}

Objective: Pneumocystis pneumonia remains a major opportunistic infection in immunocompromised patients worldwide. Colonization with Pneumocystis jirovecii has recently gained attention as an important issue for understanding the complete cycle of human Pneumocystis infection. P. jirovecii Superoxide Dismutase (SOD) gene could be a molecular target with high clinical relevance, but the epidemiological information about SOD genotypes distribution is scarce. The aim of this work was to provide information about the prevalence of genotypes of Pneumocystis SOD among Spanish patients and to describe possible differences between colonized and Pneumocystis pneumonia patients.

Methods: we developed a cross-sectional study analyzing broncho-alveolar lavage fluid samples from 30 Pneumocystis pneumonia patients, 30 colonized patients, and 20 controls using a nested PCR protocol designed to amplify the sodA gene of $P$. jirovecii. The diagnostic yield of SOD Nested PCR was evaluated against the routine practice of mtLSUrRNA Nested PCR, which is considered the gold standard.

Results: SOD locus was amplified in $90 \%$ of Pneumocystis pneumonia patients, $10 \%$ of colonized patients, and none of controls. Genotype SOD1 was observed in 11 cases (52.4\%) and genotype SOD2 in 10 cases (47.6\%). Genotype SOD2 was observed only in Pneumocystis pneumonia patients while the genotype SOD1 was observed in both colonized and Pneumocystis pneumonia patients.

Conclusions: This study provides epidemiological information about SOD genotypes distribution in Spain, showing a low genetic diversity and a predominant presence of genotype SOD1 in colonized patients. SOP Nested PCR was more sensitive and accurate assay in Pneumocystis pneumonia patients than in colonized individuals.

Keywords: Pneumocystis, molecular epidemiology, superoxide dismutase, colonization, Spain

\section{INTRODUCTION}

Pneumocystis pneumonia $(\mathrm{PcP})$ remains a major opportunistic infection in HIV-infected patients in both developed and developing countries and an emerging problem in immunocompromised patients without HIV infection worldwide (1). Today, the interest in Pneumocystis infection goes beyond PcP because a new spectrum of disease seems to emerge in immunocompetent individuals. 
The presence of Pneumocystis jirovecii in patients with underlying chronic diseases such as chronic obstructive pulmonary disease or interstitial lung diseases has been suggested to be a comorbidity factor $(2,3)$. But also, Pneumocystis colonization has gained attention as an important issue for understanding the complete cycle of human Pneumocystis infection.

For Pneumocystis infection diagnosis in humans, conventional or real-time PCR assays based on the amplification of the large subunit of mitochondrial ribosomal DNA (mtLSU rDNA) are the most commonly used, but many other sequences have been targeted. Among the most assessed sequences are the major surface glycoprotein, the small subunit of mitochondrial ribosomal DNA (mtSSU rDNA), the internal transcribed spacers, the thymidylate synthase, the dihydrofolate reductase, or the heat-shock protein (4).

Superoxide dismutases (SOD; EC 1.15.1.1) are ubiquitous key enzymes involved in the cellular defense against oxidative stress. These enzymes catalyse the first step of the detoxification of the superoxide anion in a metal cofactor dependent reaction and result essential in cellular protection against reactive oxygen species (ROS). There are several classed of SOD that have different cofactor metal present in the active site (5-7).

The gene encoding a manganese-dependent superoxide dismutase (MnSOD) has been characterized in Pneumocystis from rat, mouse, rabbit, pig, monkey, and human (8). Five genotypes have been described heretofore of which three are the most frequent: SOD1 (110C; 215T), SOD2 (110T; 215C), and SOD3 (110T, 215T) (9-11).

This gene has been used in a few epidemiological studies mainly into a multilocus approach (9-12) but the epidemiological information about SOD genotypes distribution is scarce.

The aim of this work was to provide information about the prevalence of genotypes of Pneumocystis SOD among Spanish patients and to describe possible differences between colonized and $\mathrm{PcP}$ patients.

\section{MATERIALS AND METHODS}

This study had a cross-sectional design and included 80 not selected successive male or female patients over age 18 years who were admitted to the bronchoscopy unit between 2006 and 2014 for evaluation of their disease at Virgen del Rocio University Hospital, of whom an bronchoalveolar lavage (BAL) specimen was available for analysis. Children were exclude of this study. In all cases, informed consent was obtained from the patients before obtain of BAL fluid. The study was approved by the hospital's ethics committee and it was performed according ethical principles regarding human experimentation contained in Declaration of Helsinki.

Thirty patients had AIDS-related PcP, 30 were Chronical Obstructive Pulmonary Disease (COPD) patients colonized by $P$. jirovecii without clinical or radiological signs of $\mathrm{PcP}$ and 20 were control patients with different lung diseases without Pneumocystis colonization or infection. All of them were Caucasian of Spanish origin. A single individual of control group was HIV-infected, other three were organs transplant recipients.
In all cases the presence or absence of Pneumocystis was confirmed by analyzing BAL samples with nested PCR amplification of the mtLSUrDNA gene of Pneumocystis (13). A colonized subject was defined as an individual without signs or symptoms of pneumonia from whom a respiratory sample was obtained that contained Pneumocystis-DNA detectable by PCR. A PcP patient was defined as an individual with a clinical pneumonia who had $P$. jirovecii in respiratory samples identified by microscopy or molecular methods without other causal agents.

The samples were separated into two aliquots of $250 \mu \mathrm{l}$ that were cryopreserved at $-20^{\circ} \mathrm{C}$. DNA extraction was performed with commercial Kit NucleoSpin Tissue (MachereyNagel) following the manufacturer's recommendations. Extracted DNA was eluted with $55 \mu \mathrm{l}$ of ultrapure water. Its concentration and purity (A260/A280) was determined, discarding A260/A280 values $>2$ and lower than 1.6, which would indicate contamination by proteins and RNA, respectively.

Samples that had been identified as positive by nested PCR of the mtLSUrRNA gene were further examined to study SOD gene. A nested PCR protocol was designed to amplify a locus of the sodA gene of $P$. jirovecii which included in the first amplification round, the use of the external primers MnSODFw $\left(-5^{\prime}\right.$-GGT TTA ATT AGT CTT TTA GGC AC-3') y SODR4-(5'-CCA AGA ATA ACT TTG CCT TGA G-3') to obtain a 584 bp fragment. The second round of amplification utilized the primers FS2(5'-TCT TTC TCA TGA TTT GCT TGA GG-3') and RS2-(5'CTT TCC TAT ACC TAC CAC CAC C-3') and yielded a 218 bp product. Rounds included 35 and 40 cycles of amplification, respectively. The PCR products were analyzed by electrophoresis on a $2 \%$ agarose gel containing ethidium bromide, and the bands were visualized by UV light. To prevent false positives due to contamination, pipette tips with filters were used at all stages. DNA extraction, preparation of the reaction mixture, PCR amplification, and detection were performed in different areas of the laboratory. In addition, a positive control was included in each reaction. To detect any cross-contamination, all PCR steps were performed with a negative control of sterile water. All experiments were repeated at least twice.

The products of positive nested PCR for SOD locus were purified by columns of Sephacryl S-400 (Amersham Pharmacia Biotech). Genetic characterization was performed by direct sequencing in the Genomics Service of the Institute of Biomedicine of Seville (IBiS), where they were injected into capillary sequencer ABI PRISM 3500 DNA Analyzer of Applied Biosystems.

The consensus SOD gene sequence was obtained from the NCBI web-page with the identification code and the polymorphisms were analyzed as previously described at position 110 (Y:C or T) and 215 (Y:C or T). Sequencing files were opened with Chromas lite 2.1.1 software and converted to FASTA format, in order to align the MEGA software vs. 6.0.

Finally, nested PCR for SOD assay was evaluated for sensitivity and specificity against the routine practice of Nested PCR for mtLSUrRNA gene, which is considered as gold standard. Specimens were considered true positive if they were positive by both PCR, and true negative if negative by both PCR. The diagnostic accuracy (ACC) was defined as follows: 
TABLE 1 | Characteristics of patients in study and results of the molecular typing by subgroups.

\begin{tabular}{lcccccc}
\hline Patients & $\boldsymbol{N}$ & $\begin{array}{c}\text { Sex } \\
\text { (\% males) }\end{array}$ & $\begin{array}{c}\text { Age } \\
(\overline{\boldsymbol{X}} \pm \mathrm{SD}, \\
\text { years) }\end{array}$ & $\begin{array}{c}\text { Sequenced/ } \\
\text { amplified } \\
\text { samples }\end{array}$ & $\begin{array}{c}\text { SOD1 } \\
\text { (110C; } \\
\text { 215T) }\end{array}$ & $\begin{array}{c}\text { SOD2 } \\
\text { (110T; } \\
\text { 215C) }\end{array}$ \\
\hline PCP & 30 & $73.3 \%$ & $41.12 \pm 12.05$ & $19 / 27$ & 9 & 10 \\
$\begin{array}{l}\text { COPD } \\
\text { colonized }\end{array}$ & 30 & $90.0 \%$ & $66.19 \pm 12.50$ & $2 / 3$ & 2 & 0 \\
$\begin{array}{l}\text { Non } \\
\text { colonized }\end{array}$ & 20 & $70.0 \%$ & $47.21 \pm 18.45$ & - & - & \\
\hline
\end{tabular}

$\bar{X}$, Mean; sd, standard deviation; PCP, Pneumocystis pneumonia; COPD, Chronic obstructive pulmonary disease.

$\mathrm{ACC}=(\mathrm{TP}+\mathrm{TN}) /(\mathrm{P}+\mathrm{N})$ in which $\mathrm{TP}=$ true positive; $\mathrm{TN}$ $=$ true negative; $\mathrm{P}=$ number of positive and $\mathrm{N}=$ number of negative. In addition, we calculated the Youden's J statistic (also called Youden's index) as a single statistic that captures the performance of a dichotomous diagnostic test.

\section{RESULTS}

Pneumocystis jirovecii SOD gene was detected 30 out of 80 patients, in $90 \%(27 / 30)$ of PcP patients, in 10\% (3/30) of COPD colonized patients and in none on control patients.

SOD typing was possible in 19 of the $27 \mathrm{PcP}$ patients and in two of the three COPD patients in which SOD gene was detected by PCR. Genotype SOD1 (110C; 215T) was identified in 11 cases and genotype SOD2 $(110 \mathrm{~T} ; 215 \mathrm{C})$ was identified in 10 cases (Table $\mathbf{1}$ ).

SOD Nested-PCR results were compared with data of Nested PCR for mtLSUrRNA gene, regarded as diagnostic gold standard, and concordances calculated among the results obtained. There were SOD Nested-PCR agreement with mtLSUrRNA NestedPCR in $90 \%$ of PcP cases, in $10 \%$ of COPD colonized patients, and $100 \%$ of non-infected controls.

The likelihood positive ratio, sensitivity, and specificity, Predictive Negative Value, Predictive Positive Value, diagnostic accuracy and Youden's index of SOD Nested-PCR in PcP and colonized COPD patients are showed in Table 2.

\section{DISCUSSION}

This study provides some epidemiological information about SOD genotypes distribution in Spain and shows that there are a low genetic diversity circulating in our area.

In our study, only two (SOD1 and SOD2) of the five genotypes described in the literature has been observed. These genotypes has been described as the most prevalent in several epidemiological studies $(9,10,12,14-16)$. Similarly, they are the only ones detected in other studies $(12,15)$. In this sense, the genotypic distribution of this locus in our region is similar to that described in London, UK, and Harare, Zimbabwe $(15,16)$ and differs from whose reported in France, Cuba and Portugal, where they have also detected other genotypes $(9,10,14)$. These results
TABLE 2 | Comparing characteristics and properties of SOD nested PCR as diagnostic test between PCP patients and colonized patients using mtLSU nested PCR as gold standard.

\begin{tabular}{lcc}
\hline Diagnostic variable & PcP patients & Colonized COPD patients \\
\hline Sensitivity, \% (95\% Cl) & $80 \%(62.7-90.5 \%)$ & $10 \%(3.5-25.6 \%)$ \\
Specificity, \% (95\% Cl) & $100 \%(83.9-100 \%)$ & $100 \%(83.9-100 \%)$ \\
PPV, \% (95\% Cl) & $100 \%(86.2-100 \%)$ & $100 \%(43.8-100 \%)$ \\
NPV, \%(95\% Cl) & $76.92 \%(57.9-89 \%)$ & $42.6 \%(29.5-56.7 \%)$ \\
FPR, \%(95\% Cl) & $0 \%(0-16.1 \%)$ & $0 \%(0-16.1 \%)$ \\
FNR, \%(95\% Cl) & $20 \%(9.5-37.3 \%)$ & $90 \%(74.4-96.5 \%)$ \\
Accuracy, \%(95\% Cl) & $88 \%(76.2-94.4 \%)$ & $46 \%(33-59.6 \%)$ \\
Youden's index & 0.8 & 0.1 \\
Likelihood ratio (-), & $0.2(0.1-0.41)$ & $0.9(0.8-1.1)$ \\
(95\% Cl) & &
\end{tabular}

PPV, Positive predictive value; NPV, negative predictive value; FPR, False positive rate; FNR, False negative rate; PCP, Pneumocystis pneumonia; COPD, Chronic obstructive pulmonary disease.

support the possible existence of geographic differences in the distribution of these genotypes, but further of multicentre studies covering a larger sample would be needed for confirmation.

None of the genotypes seems to be predominant over the other among the PcP patients included in our study. Nonetheless, despite appearing in only two cases, it is noteworthy that the genotype found in the colonized subjects is the SOD1 $(110 \mathrm{C}, 215 \mathrm{~T})$. This is consistent with data that suggest a potential association between this genotype with moderate or low microorganism load, which could be related with lower virulence and have clinical implications (11). However, it needs to be taken account that these are preliminary findings to be confirmed by future studies on a larger sample of patients.

In our study, differences between the values obtained for AIDS-related PcP and COPD colonized patients are probably due to different amplification rates obtained in each group due to lower parasite burden in colonized patients than in $\mathrm{PcP}$ patients (17).

The difficult to identify the SOD gene is due to it is a nuclear unicopy gene, while the mtLSU gene is located in mitochondrial ribosomes and therefore multicopy (4). This would explain why in colonized individuals where the parasite load is usually quite low, SOD gene is much more difficult to detect.

This characteristic could be useful in clinical use to distinguish colonized patients of PcP patients. However, SOP Nested PCR should not use alone for clinical diagnosis because, whilst it has a good specificity (100\%), it has a low sensitivity (80\%). Notwithstanding the foregoing, SOP Nested PCR could be used together with more sensitive PCR as those that target multicopies genes in a multiplex PCR as an early, discriminating, and accurate tool to diagnose PcP (18).

On the other hand, the lower sensitivity of SOP Nested PCR in colonized individuals (10\%) and the poor diversity of genotypes limits its usefulness in multilocus genotyping studies that include not only $\mathrm{PcP}$ patients but also colonized individuals. 
However, these conclusions need to be taken with a degree of caution as small size of study and because the information provided by a cross-sectional design is limited. Therefore, carrying out future more comprehensive studies to further define the role of SOP nested PCR in epidemiological studies of Pneumocystis infection and its utility in clinical diagnosis would be desirable.

\section{DATA AVAILABILITY STATEMENT}

The sequences of polymorphisms described in our paper can be found in Genbank under accession numbers: MG010739.1; MG010730.1.

\section{ETHICS STATEMENT}

The studies involving human participants were reviewed and approved by Comité de ética de la investigación del Hospital Universitario Virgen del Rocío. The patients/participants

\section{REFERENCES}

1. Calderón EJ, de Armas Y, Panizo MM, Wissmann G. Pneumocystis jirovecii pneumonia in Latin America. A public health problem? Expert Rev Anti Infect Ther. (2013) 11:565-70. doi: 10.1586/eri.13.41

2. Gutiérrez S, Respaldiza N, Campano E, Martínez-Risquez MT, Calderón EJ, De la Horra C. Pneumocystis jirovecii colonization in chronic pulmonary disease. Parasite. (2011) 18:121-6. doi: 10.1051/parasite/2011182121

3. Martínez-Rísquez MT, Friaza V, de la Horra C, Martín-Juan J, Calderón EJ, Medrano FJ. Pneumocystis jirovecii infection in patients with acute interstitial pneumonia. Rev Clin Esp. (2018) 218:417-20. doi: 10.1016/j.rceng.2018. 04.013

4. Calderón EJ, Gutiérrez-Rivero S, Durand-Joly I, Dei-Cas E. Pneumocystis infection in humans: diagnosis and treatment. Expert Rev Anti Infect Ther. (2010) 8:683-701. doi: 10.1586/eri.10.42

5. Valko M, Leibfritz D, Moncol J, Cronin MT, Mazur M, Telser J. Free radicals and antioxidants in normal physiological functions and human disease. Int J Biochem Cell Biol. (2007) 39:44-84. doi: 10.1016/j.biocel.2006.07.001

6. Brioukhanov AL, Netrusov AI, Eggen RI. The catalase and superoxide dismutase genes are transcriptionally up-regulated upon oxidative stress in the strictly anaerobic archaeon Methanosarcina barkeri. Microbiology. (2006) 152:1671-7. doi: 10.1099/mic.0.28542-0

7. Zeinali F, Homaei A, Kamrani E. Sources of marine superoxide dismutases: characteristics and applications. Int J Biol Macromol. (2015) 79:627-37. doi: 10.1016/j.ijbiomac.2015.05.053

8. Denis CM, Mazars E, Guyot K, Odberg-Ferragut C, Viscogliosi E, Dei-Cas E, et al. Genetic divergence at the SODA locus of six different formae speciales of Pneumocystis carinii. Med Mycol. (2000) 38:289-300. doi: 10.1080/7140 30952

9. Maitte C, Leterrier M, Le Pape P, Miegeville M, Morio F. Multilocus sequence typing of Pneumocystis jirovecii from clinical samples: how many and which loci should be used? J Clin Microbiol. (2013) 51:2843-9. doi: 10.1128/JCM.01073-13

10. Monroy-Vaca EX, de Armas Y, Illnait-Zaragozí MT, Diaz R, Toraño G, Vega D, et al. Genetic diversity of Pneumocystis jirovecii in colonized Cuban infants and toddlers. Infect Genet Evolut. (2014) 22:60-6. doi: 10.1016/j.meegid.2013.12.024

11. Esteves F, Gaspar J, de Sousa B, Antunes F, Mansinho K, Matos O. Pneumocystis jirovecii multilocus genotyping in pooled DNA samples: a new approach for clinical and epidemiological studies. Clin Microbiol Infect. (2012) 18:E177-84. doi: 10.1111/j.1469-0691.2012.03828.x provided their written informed consent to participate in this study.

\section{AUTHOR CONTRIBUTIONS}

EC and $\mathrm{CH}$ conceived and designed the research. AG-M and VF collected and analyzed the data. YA and FM performed the statistical analysis. VF, AG-M, CH, and FM contributed to the development of the study and interpreted the data. RM and EC wrote the draft of the manuscript. All authors reviewed and approved the final version of the manuscript.

\section{FUNDING}

This study was supported, in part, by the Red Iberoamericana sobre Pneumocystosis in the framework of The Ibero-American Programme for Science, Technology and Development (grant CYTED 212RT0450).
12. Esteves F, Gaspar J, De Sousa B, Antunes F, Mansinho K, Matos O. Clinical relevance of multiple single-nucleotide polymorphisms in Pneumocystis jirovecii Pneumonia: development of a multiplex PCR-single-base-extension methodology. J Clin Microbiol. (2011) 49:1810-5. doi: 10.1128/JCM.02303-10

13. Pederiva MA, Wissmann G, Friaza V, Morilla R, de La Horra C, Montes-Cano MA, et al. High prevalence of Pneumocystis jirovecii colonization in Brazilian cystic fibrosis patients. Med Mycol. (2012) 50:556-60. doi: $10.3109 / 13693786.2011 .645892$

14. Esteves F, Gaspar J, Tavares A, Moser I, Antunes F, Mansinho K, et al. Population structure of Pneumocystis jirovecii isolated from immunodeficiency virus-positive patients. Infect Genet Evolut. (2010) 10:192-9. doi: 10.1016/j.meegid.2009.12.007

15. Wakefield AE, Lindley AR, Ambrose HE, Denis CM, Miller RF. Limited asymptomatic carriage of Pneumocystis jiroveci in human immunodeficiency virus-infected patients. J Infect Dis. (2003) 187:901-8. doi: 10.1086/368165

16. Miller RF, Lindley AR, Malin AS, Ambrose HE, Wakefield AE. Isolates of Pneumocystis jirovecii from Harare show high genotypic similarity to isolates from London at the superoxide dismutase locus. Trans $R$ Soc Trop Med Hyg. (2005) 99:202-6. doi: 10.1016/j.trstmh.2004.09.005

17. Fauchier T, Hasseine L, Gari-Toussaint M, Casanova V, Marty PM, Pomares C. Detection of Pneumocystis jirovecii by quantitative PCR to differentiate colonization and pneumonia in immunocompromised HIVpositive and HIV-negative patients. J Clin Microbiol. (2016) 54:1487-95. doi: 10.1128/JCM.03174-15

18. Montesinos I, Delforge ML, Ajjaham F, Brancart F, Hites M, Jacobs F, et al. Evaluation of a new commercial real-time PCR assay for diagnosis of Pneumocystis jirovecii pneumonia and identification of dihydropteroate synthase (DHPS) mutations. Diagn Microbiol Infect Dis. (2017) 87:32-6. doi: 10.1016/j.diagmicrobio.2016.10.005

Conflict of Interest: The authors declare that the research was conducted in the absence of any commercial or financial relationships that could be construed as a potential conflict of interest.

Copyright (c) 2019 Morilla, González-Magaña, Friaza, de Armas, Medrano, Calderón and de la Horra. This is an open-access article distributed under the terms of the Creative Commons Attribution License (CC BY). The use, distribution or reproduction in other forums is permitted, provided the original author $(s)$ and the copyright owner(s) are credited and that the original publication in this journal is cited, in accordance with accepted academic practice. No use, distribution or reproduction is permitted which does not comply with these terms. 